AMES-HET-03-06

BNL-HET-03/19

\title{
Three heavy jet events at hadron colliders as a sensitive probe of the Higgs sector
}

\author{
David Atwood* \\ Department of Physics and Astronomy, Iowa State University, Ames, IA 50011, USA \\ Shaouly Bar-Shalon and Gad Eilant \\ Physics Department, Technion-Institute of Technology, Haifa 32000, Israel \\ Amarjit Son $\$$ \\ Theory Group, Brookhaven National Laboratory, Upton, NY 11973, USA
}

(Dated: October 22, 2018)

\begin{abstract}
Assuming that a non-standard neutral Higgs with an enhanced Yukawa coupling to a bottom quark is observed at future hadron experiments, we propose a method for a better understanding of the Higgs sector. Our procedure is based on "counting" the number of events with heavy jets (where "heavy" stands for a $c$ or $b$ jet) versus $b$ jets, in the final state of processes in which the Higgs is produced in association with a single high $p_{T} c$ or $b$ jet. We show that an observed signal of the type proposed, at either the Tevatron or the LHC, will rule out the popular two Higgs doublet model of type II as well as its supersymmeric version - the Minimal Supersymmetric Standard Model (MSSM), and may provide new evidence in favor of some more exotic multi Higgs scenarios. As an example, we show that in a version of a two Higgs doublet model which naturally accounts for the large mass of the top quark, our signal can be easily detected at the LHC within that framework. We also find that such a signal may be observable at the upgraded Tevatron RunIII, if the neutral Higgs in this model has a mass around $100 \mathrm{GeV}$ and $\tan \beta \gtrsim 50$ and if the efficiency for distinguishing a $c$ jet from a light jet reaches the level of $\sim 50 \%$.
\end{abstract}

PACS numbers: 12.15.Ji, 12.60.Fr, 12.60.-i, 14.80.Cp

\section{INTRODUCTION}

With the Tevatron RunII underway at Fermilab, the future Tevatron upgrade RunIII and the Large Hadron Collider (LHC) at CERN, the discovery of the Higgs boson is currently believed to be "around the corner". These machines will be able to detect the neutral Higgs over its entire theoretically allowed mass range [1].

If the Higgs Yukawa coupling to a bottom quark is enhanced, as predicted for example in general multi Higgs models and in the supersymmetric version of the two Higgs Doublet Model (2HDM) - the MSSM - when tan $\beta$ is large, then the neutral Higgs may be produced at Leading Order (LO) through $b \bar{b}$ fusion $b \bar{b} \rightarrow h$ [2]. In such models, a sizable production rate of a neutral Higgs in association with $b$ quark jets is also expected via the subprocesses $g b \rightarrow b h[3,4]$ and $g g, q \bar{q} \rightarrow b \bar{b} h$ [5]. ${ }^{[1]}$ In particular, as was shown in [3], if one demands that only one $b$ jet will be produced (in association with the Higgs) at high $p_{T}$, then $g b \rightarrow b h$ becomes the LO and is therefore the dominant Higgs-bottom associated production mechanism for large $\tan \beta$. For example, $g b \rightarrow b h$ followed by $h \rightarrow b \bar{b}$ may already prove to be a useful probe of the

\footnotetext{
*Electronic address: atwood@iastate.edu

${ }^{\dagger}$ Electronic address: shaouly@physics.technion.ac.il

¥Electronic address: eilam@physics.technion.ac.il

$\S$ Electronic address: soni@bnl.gov

[1] Unless stated otherwise, $h$ will be used to denote the neutral Higgs that drives the processes under consideration.
}

neutral Higgs sector at the Tevatron future runs [6].

In this work we assume that indeed the Higgs has an enhanced coupling to bottom quarks and that it will be therefore discovered or observed in association with bottom quarks jets, via one of the above production channels. Discovery of such a non-standard Higgs should start an exploration era of the various Higgs Yukawa couplings to fermions in order to decipher the origin and detailed properties of the Higgs sector. For that purpose, special observables should be devised that will enable the experimentalists to pin-down some specific Higgs Yukawa interactions. In particular, one should find observables which are sensitive to a specific Yukawa coupling or combination of couplings, and are therefore able to exclude models with an enhanced Higgs-bottom Yukawa vertex, or provide further evidence in favor of a definite Higgs scenario.

In this paper we follow this line of thought and present a method which is useful for an investigation of the relation between the Higgs-charm and Higgs-bottom Yukawa couplings. We focus on neutral Higgs production via a single high $p_{T}$ charm or bottom jet, $q g \rightarrow q h, q=c$ or $b$, followed by $h \rightarrow b \bar{b}$ or $h \rightarrow c \bar{c}^{[2]}$ Our final state is therefore defined to be composed out of exactly three non-light jets, where a light jet means a $u, d, s$ or $g$ (gluon) jet. If the Higgs is not the Standard Model (SM) one, par-

[2] We will not explicitly write the charge of the quarks involved. All charge-conjugate sub-processes are understood throughout this paper. 
ticularly if the Higgs-bottom Yukawa is large, then this class of processes may play an important role in Higgs searches, discovery and characterization at hadron colliders.

Based on this type of production mechanism, we suggest an observable which is particularly sensitive to new physics in the ratio between the Higgs-charm and the Higgs-bottom Yukawa couplings. We find that our signal is insensitive to the Higgs sectors of the type II 2HDM (2HDMII) and of the MSSM. Therefore, a positive signal of our observable at either the Tevatron or the LHC will rule out these models. We also consider a different type of a $2 \mathrm{HDM}$, the so called "2HDM for the top quark" (T2HDM), which is an interesting model designed to naturally accommodate the large mass of the top quark. This model may be viewed as an effective low energy theory wherein the Yukawa interactions mimic some high energy dynamics which generates both the top mass and the weak scale. The two scalar doublets could be composite, as in top-color models, and the Yukawa interactions could be the residual effect of some higher energy four-Fermi operators. Interestingly, for this T2HDM our signal is significantly enhanced. We thus show that if this version of a $2 \mathrm{HDM}$ underlies the Higgs sector, then our signal will be easily detected (to many standard deviations) at the LHC and may also be detected at the Tevatron if $\tan \beta$ is large enough and if the efficiency of tagging a $c$ jet as a heavy jet is of $\mathcal{O}(50 \%)$.

The paper is organized as follows: in section II we define our signal. In section III we present the numerical setup and discuss the experimental background. In section IV we evaluate our signal in three different versions of a $2 \mathrm{HDM}$ and discuss the expectations from the T2HDM, the 2HDMII and the MSSM. In section V we summarize our results.

\section{NOTATION AND DEFINITION OF THE SIGNAL}

Let $j_{h}$ denote a "heavy" $b$ or $c$-quark jet. Specifically, a $j_{h}$ jet should not be a $u, d, s$ or gluon jet (i.e., with the veto $j_{h} \neq u, d, s, g$ jet). Let $j_{b}$ be a $b$-quark jet. Clearly, every jet of type $j_{b}$ is also a $j_{h}$. Likewise, let us denote by $j_{c}$ a $c$-quark jet.

We thus define the cross-sections

$$
\begin{aligned}
\sigma_{j_{h} j_{h} j_{h}} & \equiv \sigma\left(p p \text { or } p \bar{p} \rightarrow j_{h} j_{h} j_{h}+X\right) \\
\sigma_{j_{b} j_{b} j_{b}} & \equiv \sigma\left(p p \text { or } p \bar{p} \rightarrow j_{b} j_{b} j_{b}+X\right)
\end{aligned}
$$

and the ratio

$$
\mathcal{R} \equiv \frac{\sigma_{j_{h} j_{h} j_{h}}}{\sigma_{j_{b} j_{b} j_{b}}}
$$

Note that, since $j_{h}$ is either a $j_{b}$ or a $j_{c}$, it follows that:

$$
\sigma_{j_{h} j_{h} j_{h}} \equiv \sigma_{j_{b} j_{b} j_{b}}+\sigma_{j_{c} j_{b} j_{b}}+\sigma_{j_{b} j_{c} j_{c}}+\sigma_{j_{c} j_{c} j_{c}} .
$$

From the above definitions it is evident that:

$$
\mathcal{R}-1 \propto \frac{Y_{c}^{2}}{Y_{b}^{2}}+\mathcal{O}\left(\frac{Y_{c}^{4}}{Y_{b}^{4}}\right) .
$$

where $Y_{c}$ and $Y_{b}$ are the Higgs-charm and Higgs-bottom Yukawa couplings, respectively.

In reality, one has to include non-ideal efficiencies for jet identification. Therefore, we define the quantity $\epsilon_{j}^{k}$ to be the efficiency for identifying a $j$ jet as a $k$ jet. Thus, for example, $\epsilon_{l}^{b}$ or $\epsilon_{l}^{h}$ are the efficiencies for misidentifying a light jet $\left(j_{l}\right)$ as a $b$ jet $\left(j_{b}\right)$ or as a heavy jet $\left(j_{h}\right)$, respectively. Using these efficiency factors we define the experimental observable as:

$$
R^{M} \equiv \frac{\bar{\sigma}_{j_{h} j_{h} j_{h}}^{M}}{\bar{\sigma}_{j_{b} j_{b} j_{b}}^{M}}
$$

where $R=\bar{\sigma}_{j_{h} j_{h} j_{h}} / \bar{\sigma}_{j_{b} j_{b} j_{b}}$ with $\bar{\sigma}_{j_{h} j_{h} j_{h}}, \bar{\sigma}_{j_{b} j_{b} j_{b}}$ being the effective signals for cross-sections that will actually be measured in the experiment and $R^{M}$ is the value of $R$ calculated within a specific Higgs model $M$ via the subprocesses $g q \rightarrow q h \rightarrow q q q, q=c$ or $b$. Thus, in the limit of an ideal detector where $\epsilon_{j}^{k}=\delta_{j}^{k}, R^{M}=\mathcal{R}^{M}$ (where $\mathcal{R}^{M}$ means the "ideal" ratio defined in (3) calculated within model M). In particular, these cross-sections include all tagging efficiencies as follows:

$$
\begin{aligned}
\bar{\sigma}_{j_{b} j_{b} j_{b}}^{M} & =\sum_{j_{1}, j_{2}, j_{3}} \epsilon_{j_{1}}^{b} \epsilon_{j_{2}}^{b} \epsilon_{j_{3}}^{b} \times \sigma_{j_{1} j_{2} j_{3}}^{M}, \\
\bar{\sigma}_{j_{h} j_{h} j_{h}}^{M} & =\sum_{j_{1}, j_{2}, j_{3}} \epsilon_{j_{1}}^{h} \epsilon_{j_{2}}^{h} \epsilon_{j_{3}}^{h} \times \sigma_{j_{1} j_{2} j_{3}}^{M} .
\end{aligned}
$$

In the limit $\left(Y_{c} / Y_{b}\right)^{2} \rightarrow 0$, one obtains

$\left.R^{M} \rightarrow R^{0} \equiv \frac{\bar{\sigma}_{j_{h} j_{j_{h} j_{h}}}^{M}}{\bar{\sigma}_{j_{b} j_{b} j_{b}}^{M}}\right|_{\left(\frac{Y_{c}}{Y_{b}}\right)^{2} \rightarrow 0} \rightarrow \frac{\left(\epsilon_{b}^{h}\right)^{3} \sigma_{j_{b} j_{b} j_{b}}^{M}}{\left(\epsilon_{b}^{b}\right)^{3} \sigma_{j_{b} j_{b} j_{b}}^{j_{b}}}=\left(\frac{\epsilon_{b}^{h}}{\epsilon_{b}^{b}}\right)^{3}$.

Therefore, due to the non-ideal efficiencies (in particular if $\epsilon_{b}^{h} \neq \epsilon_{b}^{b}$ ), we have $R^{0} \neq 1$ even if the Higgs Yukawa coupling to the charm quark is negligible. As will be shown below, this limit is applicable to a neutral Higgs either of MSSM or of $2 \mathrm{HDMII}$ origin, if $\tan \beta$ is large. That is, for large $\tan \beta$

$$
R^{M S S M}, R^{2 H D M I I} \rightarrow R^{0}=\left(\frac{\epsilon_{b}^{h}}{\epsilon_{b}^{b}}\right)^{3} .
$$

A deviation from $R^{M}=R^{0}$, indicating a high rate for $h \rightarrow c \bar{c}$, signals a specific type of new physics in the Higgs 
sector, e.g., beyond the MSSM or the 2HDMII Higgs scenario. Such a deviation is parameterized by:

$$
\delta R^{M}=R^{M}-R^{0} \propto\left(\frac{Y_{c}}{Y_{b}}\right)^{2}+\mathcal{O}\left(\frac{Y_{c}^{4}}{Y_{b}^{4}}\right) .
$$

The significance with which $\delta R^{M}$ can be measured depends on the experimental error $\delta R_{\exp }^{M}$ :

$$
\delta R_{\exp }^{M}=R^{M} \sqrt{\frac{\left(\Delta N_{j_{h} j_{h} j_{h}}\right)^{2}}{\left(N_{j_{h} j_{h} j_{h}}^{M}\right)^{2}}+\frac{\left(\Delta N_{j_{b} j_{b} j_{b}}\right)^{2}}{\left(N_{j_{b} j_{b} j_{b}}^{M}\right)^{2}}},
$$

where $N_{j_{h} j_{h} j_{h}}^{M}=\bar{\sigma}_{j_{h} j_{h} j_{h}}^{M} \times L$ and similarly for $N_{j_{b} j_{b} j_{b}}^{M}$, with $L$ being the integrated luminosity at the given collider. The errors in the measurements of $N_{j_{h} j_{h} j_{h}}^{M}$ and $N_{j_{b} j_{b} j_{b}}^{M}$ are (statistical only): $\Delta N_{j_{h} j_{h} j_{h}}=\sqrt{N_{j_{h} j_{h} j_{h}}}$ and $\Delta N_{j_{b} j_{b} j_{b}}=\sqrt{N_{j_{b} j_{b} j_{b}}}$, where $N_{j_{h} j_{h} j_{h}}$ and $N_{j_{b} j_{b} j_{b}}$ (i.e., without the superscript $\mathrm{M}$ ) are the total number of events dominated by the background processes.

Eq.(12) can be simplified to:

$$
\delta R_{\exp }^{M}=\frac{\sqrt{N_{j_{h} j_{h} j_{h}}+\left(R^{M}\right)^{2} N_{j_{b} j_{b} j_{b}}}}{N_{j_{b} j_{b} j_{b}}^{M}},
$$

therefore, since $N_{j_{b} j_{b} j_{b}}^{M} \propto Y_{b}^{2}$, it follows that $\delta R_{\exp }^{M} \propto$ $1 / Y_{b}^{2}$. In particular, if $Y_{b} \propto \tan \beta$, then $\delta R_{\exp }^{M} \propto$ $1 / \tan ^{2} \beta$.

The condition, $\delta R^{M}>\delta R_{\exp }^{M}$ signals that new physics in the ratio between the $h c c$ and $h b b$ Yukawa couplings can be seen, with a statistical significance of

$$
N_{S D}=\frac{\delta R^{M}}{\delta R_{\exp }^{M}}
$$

\section{NUMERICAL SETUP AND BACKGROUND}

Before presenting our results let us describe the numerical setup:

- All signal and background cross-sections are calculated at leading order (tree-level), using the COMPHEP package 7] with the CTEQ5L [8] parton distribution functions.

- Throughout our entire analysis to follow, the following set of cuts are employed in both signal and background cross-sections:

1. For both the Tevatron and the LHC, in order for the jets to be within the tagging region of the silicon vertex detector, we require all three jets to have the following minimum transverse momentum $\left(p_{T}\right)$ and rapidity $(\eta)$ coverages:
Tevatron: $p_{T}(j)>15 \mathrm{GeV},|\eta(j)|<2$, LHC: $\quad p_{T}(j)>30 \mathrm{GeV},|\eta(j)|<2.5$, where $j$ is any of the three jets in the final state.

2. The signal cross-sections always have one pair of jets (coming from the Higgs decay) that should reconstruct the Higgs mass. Thus, in order to improve the signal to background ratio, we require for both the signal and background cross-sections that only one jet pair (out of the three possible jet pairs in the final state), will have an invariant mass within $m_{h} \pm 0.05 m_{h}$, i.e., within $\pm 5 \%$ of $m_{h}$ around $m_{h}$. That is, we reject events if the invariant masses of more than one $j_{i} j_{k}$ pair are within $m_{h}-0.05 m_{h}<M_{j_{i} j_{k}}<m_{h}+0.05 m_{h}$. This acceptance cut is found to significantly improve the signal to background ratio.

3. We impose a common lower cut on the invariant masses of all three jet pairs: $M_{j_{i} j_{k}}>$ $m_{h} / 2$. This additional cut further improves the signal to background ratio.

4. We require the final state partons to be separated by a cone angle of $\Delta R>0.7$, where here $\Delta R$ stands for $\sqrt{\Delta \eta^{2}+\Delta \varphi^{2}}$.

- We use the $\overline{M S}$ running Yukawa couplings $\bar{Y}_{b}\left(\mu_{R}\right) \propto\left(\bar{m}_{b}\left(\mu_{R}\right) / v\right), \bar{Y}_{c}\left(\mu_{R}\right) \propto\left(\bar{m}_{c}\left(\mu_{R}\right) / v\right)$, where $\bar{m}_{b}\left(\mu_{R}\right)$ and $\bar{m}_{c}\left(\mu_{R}\right)$ are $\overline{M S}$ running masses. We take a renormalization scale of $\mu_{R}=m_{h}$ as our central value. In particular, $\bar{m}_{b}\left(m_{h}\right)$ and $\bar{m}_{c}\left(m_{h}\right)$ are calculated via the next-to-leading-order heavy quark $\overline{M S}$ running mass equation [9], using $\bar{m}_{b}\left(\bar{m}_{b}\right)$ and $\bar{m}_{c}\left(\bar{m}_{c}\right)$ as the initial conditions. This brings up some uncertainty corresponding to the allowed range of $\bar{m}_{b}\left(\bar{m}_{b}\right)$ and $\bar{m}_{c}\left(\bar{m}_{c}\right): 4 \mathrm{GeV}<\bar{m}_{b}\left(\bar{m}_{b}\right)<$ $4.5 \mathrm{GeV}$ and $1 \mathrm{GeV}<\bar{m}_{c}\left(\bar{m}_{c}\right)<1.4 \mathrm{GeV}$, see [10. In what follows we use $\bar{m}_{b}\left(\bar{m}_{b}\right)=4.26 \mathrm{GeV}$ and $\bar{m}_{c}\left(\bar{m}_{c}\right)=1.26 \mathrm{GeV}$ as our central values [10], which give the $\bar{m}_{b}\left(m_{h}\right)$ and $\bar{m}_{c}\left(m_{h}\right)$ masses listed in Table 【

TABLE I: Running charm and bottom quark $\overline{M S}$ (NLO)

\begin{tabular}{|c|c|c|c|}
\hline$\Downarrow[\mathrm{GeV}] \Rightarrow$ & $\mu_{R}=100$ & $\mu_{R}=120$ & $\mu_{R}=140$ \\
\hline $\bar{m}_{b}\left(\mu_{R}\right)$ & 2.94 & 2.89 & 2.86 \\
\hline $\bar{m}_{c}\left(\mu_{R}\right)$ & 0.61 & 0.6 & 0.59 \\
\hline
\end{tabular}
masses at $\mu_{R}=m_{h}$ for $m_{h}=100,120$ and $140 \mathrm{GeV}$, with the initial conditions $\bar{m}_{b}\left(\bar{m}_{b}\right)=4.26 \mathrm{GeV}$ and $\bar{m}_{c}\left(\bar{m}_{c}\right)=1.26$ $\mathrm{GeV}$.

At some instances we will refer to the "maximal signal" possible. This means that we are evaluating the signal cross-sections with the initial conditions $\bar{m}_{b}\left(\bar{m}_{b}\right)=4.5 \mathrm{GeV}$ and $\bar{m}_{c}\left(\bar{m}_{c}\right)=1.4$ 
$\mathrm{GeV}$, i.e., at their upper allowed values, which at $\mu_{R}=100 \mathrm{GeV}$ gives: $\bar{m}_{b}(100 \mathrm{GeV})=3.1 \mathrm{GeV}$ and $\bar{m}_{c}(100 \mathrm{GeV})=0.68 \mathrm{GeV}$.

- Unless stated otherwise, the parton distribution functions are evaluated with a factorization scale of $\mu_{F}=m_{h}$. Thus, we use a common factorization and renormalization scale $\mu=\mu_{F}=\mu_{R}$ and take $\mu=m_{h}$ as our nominal value. The uncertainty of our signal cross-sections, obtained by varying the factorization scale $\mu_{F}$ about its central value $\left(\mu_{F}=m_{h}\right)$ within the range $m_{h} / 4<\mu_{F}<2 m_{h}$, will also be investigated.

- We do not consider the next-to-leading-order (NLO) contributions to $\sigma(q g \rightarrow q h)$. As was shown in 3], the NLO contribution to $\sigma(b g \rightarrow b h)$ can amount to an effective $K$-factor of about 1.5 at the Tevatron and about 1.2 at the LHC. Assuming that a similar $K$-factor applies also to $\sigma(\mathrm{cg} \rightarrow$ $c h$ ), our signal $R^{M}$ is essentially insensitive to the NLO corrections since it involves ratios of these cross-sections. Moreover, $\delta R_{\exp }^{M}$ is proportional to $\sqrt{K^{B}} / K^{S}$, where $K^{B}$ and $K^{S}$ are the $K$-factors for the background and the signal three jets events, respectively. Thus, at NLO, the statistical significance of our signal $\left(N_{S D}=\delta R^{M} / \delta R_{\text {exp }}^{M}\right)$ should be about a factor of $K^{S} / \sqrt{K^{B}}$ larger or smaller than our tree-level estimate, depending on the size of $K^{B}$ and $K^{S}$. For $K^{B} \sim K^{S}>1$, our tree-level prediction for $N_{S D}$ is on the conservative side.

- Since we require our signal to be composed out of events with exactly three jets in the final state (i.e., 3 heavy jets, $j_{h} j_{h} j_{h}$ ), we do not consider processes with 4 heavy jets or $4 b$ jets in the final state, e.g., $g g, q \bar{q} \rightarrow b \bar{b} h \rightarrow b \bar{b} b \bar{b}$. These type of sub-processes constitute a partial source of the $K$ factor for the 3 heavy jets signal cross-sections [3]. Moreover, as was further demonstrated in 3] for the case of Higgs-bottom associated production at hadron colliders, even if we had relaxed our demand for $e x$ actly 3 jets in the final state to allow for 3 or 4 jets with 3 or 4 jet tags, the contribution of events with 4 jets to $R^{M}$ would still constitute no more than $10 \%$ of those with exactly 3 jets in the final state.

Let us now discuss the background cross-sections. The irreducible background for the various three heavy jet final states, $j_{h} j_{h} j_{h}$ with $h=c$ or $b$, is dominated by the QCD sub-processes $b g \rightarrow b g \rightarrow b b b, b c c$ and $c g \rightarrow c g \rightarrow$ $c c c, c b b$. Other processes like $Z$ production followed by its decay, $b g \rightarrow b Z \rightarrow b b b, b c c$ and $c g \rightarrow c Z \rightarrow c c c, c b b$, and QED also contribute to the background. In what follows, this irreducible background is calculated using the COMPHEP package [7, where all possible tree-level diagrams (QCD, Electroweak and QED) for the $j_{h} j_{h} j_{h}$ final state are included. In addition, due to the non-ideal efficiencies, e.g., the non-zero probability of misidentifying a light jet for a heavy jet, there is a reducible background for the three heavy jet final states coming from sub-processes in which one, two or all three jets are light. Since one expects that $\epsilon_{l}^{h}, \epsilon_{l}^{b} \ll \epsilon_{b}^{h}, \epsilon_{b}^{b}, \epsilon_{c}^{h}, \epsilon_{c}^{b}$ (see e.g., [1] ), the reducible background cross-sections, when multiplied by $\epsilon_{l}^{h}$ or $\epsilon_{l}^{b}$ are dominated by processes in which only one jet out of the three is light. Moreover, we find that, at both the Tevatron and the LHC, the one-light-jet reducible background is by far controlled by the $g g \rightarrow g b b$ and $g g \rightarrow g c c$ sub-processes, which will be therefore included in our background estimation. For example, the contribution of $g g \rightarrow g b b$ to the $j_{h} j_{h} j_{h}$ final state is: $\bar{\sigma}_{j_{h} j_{h} j_{h}}^{B}=\sigma_{g g \rightarrow g b b} \times \epsilon_{l}^{h}\left(\epsilon_{b}^{h}\right)^{2}$ (the background signals will always be denoted by the superscript $B)$.

In Tables II and III we list the background crosssections at the Tevatron and at the LHC, respectively, where all cross-sections are calculated with the set of cuts 1-4, described earlier. The background cross-sections are calculated with a factorization scale set to $\mu_{F}=\sqrt{\hat{s}}$, where $\hat{s}$ is the square of the c.m. energy of the hard cross-sections.

TABLE II: Background cross-sections in [fb], for the Tevatron with a c.m. of $\sqrt{s}=2 \mathrm{TeV}$. All cross-sections are calculated with the following kinematical cuts (some defined as a function of $m_{h}$ ): (1) $p_{T}(j)>15 \mathrm{GeV}$, (2) $\left|\eta_{j}\right|<2$, where $j$ is any (heavy and light) of the three jets in the final state, (3) the acceptance cut that the invariant mass of only one jet pair $M_{j_{i} j_{k}}, i \neq k$, out of the three possible pairs of jets in the final state is within the mass range $\left(m_{h}-0.05 m_{h}\right)<M_{j_{i} j_{k}}<\left(m_{h}+0.05 m_{h}\right)$, (4) $M_{j_{i} j_{k}}>m_{h} / 2$, for all three jet pairs, and (5) any two partons in the final state are separated by a cone of $\Delta R>0.7$. Numbers are rounded to the $1 \%$ accuracy as was obtained from the COMPHEP numerical sessions.

\begin{tabular}{|c||c|c|c|}
\hline \multicolumn{4}{|c|}{ Background cross-sections at the Tevatron } \\
\hline \multirow{2}{*}[\mathrm{fb}]{$\Downarrow /[\mathrm{GeV}] \Rightarrow$} & $m_{h}$ used for the kinematical cuts \\
\cline { 2 - 4 }$m_{h}=100$ & $m_{h}=120$ & $m_{h}=140$ \\
\hline \hline$\sigma^{B}(g b \rightarrow b b b$ or $b c c)$ & $4.4 \cdot 10^{3}$ & $1.8 \cdot 10^{3}$ & $9.5 \cdot 10^{2}$ \\
$\sigma^{B}(g c \rightarrow c b b$ or $c c c)$ & $7.2 \cdot 10^{3}$ & $3.0 \cdot 10^{3}$ & $16.3 \cdot 10^{2}$ \\
$\sigma^{B}(g g \rightarrow g b b$ or $g c c)$ & $230 \cdot 10^{3}$ & $94 \cdot 10^{3}$ & $41 \cdot 10^{3}$ \\
\hline
\end{tabular}

TABLE III: Background cross-sections in [fb], for the LHC with a c.m. of $\sqrt{s}=14 \mathrm{TeV}$. All cross-sections are calculated with $p_{T}(j)>30 \mathrm{GeV},\left|\eta_{j}\right|<2.5$ and the additional kinematical cuts 3-5 as in Table (see caption to Table II).

\begin{tabular}{|r||r|r|r|}
\hline \multicolumn{5}{|c|}{ Background cross-sections at the LHC } \\
\hline \multirow{2}{*}[\mathrm{fb}]{$\Downarrow /[\mathrm{GeV}] \Rightarrow$} & $m_{h}$ used for the kinematical cuts \\
\cline { 2 - 4 } & $m_{h}=100$ & $m_{h}=120$ & $m_{h}=140$ \\
\hline \hline$\sigma^{B}(g b \rightarrow b b b$ or $b c c)$ & $133 \cdot 10^{3}$ & $111 \cdot 10^{3}$ & $84 \cdot 10^{3}$ \\
$\sigma^{B}(g c \rightarrow c b b$ or $c c c)$ & $190 \cdot 10^{3}$ & $159 \cdot 10^{3}$ & $120 \cdot 10^{3}$ \\
$\sigma^{B}(g g \rightarrow g b b$ or $g c c)$ & $5680 \cdot 10^{3}$ & $4720 \cdot 10^{3}$ & $3540 \cdot 10^{3}$ \\
\hline
\end{tabular}




\section{EXPECTATIONS FROM TWO HIGGS DOUBLET MODELS}

The signal cross-sections depend on the Higgs couplings and therefore on the underlying Higgs model. In what follows we will investigate the sensitivity of our signal $R^{M}$ to various versions of a $2 \mathrm{HDM}$.

The most general 2HDM Yukawa term is given by

$$
\begin{aligned}
\mathcal{L}_{Y}= & -\sum_{i, j} \bar{Q}_{L}^{i}\left[\left(U_{i j}^{1} \tilde{\Phi}_{1}+U_{i j}^{2} \tilde{\Phi}_{2}\right) u_{R}^{j}\right. \\
& \left.+\left(D_{i j}^{1} \Phi_{1}+D_{i j}^{2} \Phi_{2}\right) d_{R}^{j}\right]
\end{aligned}
$$

where $Q_{L}$ is the $\mathrm{SU}(2)$ left-handed quark doublet, $u_{R}$ and $d_{R}$ are the right-handed up and down quark $\mathrm{SU}(2)$ singlets, respectively, and $\tilde{\Phi}_{1,2}=i \sigma_{2} \Phi_{1,2}^{*}$. Also, $U^{1}, U^{2}, D^{1}, D^{2}$ are general Yukawa $3 \times 3$ matrices in flavor space. The different types of 2HDM's are then categorized according to the different choices of the Yukawa matrices $U^{1}, U^{2}, D^{1}, D^{2}$.

\section{A. The 2HDM for the top quark - T2HDM}

As an example of a Higgs sector that can give rise to an observable effect via $R^{M}$, we consider first the so called "2HDM for the top", in which the top quark receives a special status [12]. We will denote this model by T2HDM. In the T2HDM one defines [12]

$$
\begin{aligned}
U_{i j}^{1} & \rightarrow G_{i j} \times\left(\delta_{j 1}+\delta_{j 2}\right), \\
U_{i j}^{2} & \rightarrow G_{i j} \times \delta_{j 3} \\
D_{i j}^{2} & \rightarrow 0
\end{aligned}
$$

where $G$ is an unknown Yukawa $3 \times 3$ matrix in quark flavor space. The large mass of the top is, thus, naturally accommodated in the T2HDM by coupling the second Higgs doublet $\left(\Phi_{2}\right)$, which has a much larger Vacuum Expectation Value (VEV), only to the top quark. This model has therefore large $\tan \beta=v_{2} / v_{1}$ by construction. That is, $v_{2}$ is the VEV responsible for the top quark mass, while $v_{1}$ generates the masses of all the other quarks.

Like any other 2HDM, the Higgs spectrum of the T2HDM is composed out of three neutral Higgs; two CPeven scalars and one pseudoscalar, and a charged Higgs. Choosing the basis $\alpha=\beta$, where $\alpha$ is the usual mixing angle between the two CP-even Higgs particles (see e.g., [13]), the neutral Higgs spectrum of the T2HDM is rotated such that it contains a SM-like neutral Higgs, $H_{S M}$, (with the SM couplings to fermions), a CP-even Higgs, $h$, and a pseudoscalar (CP-odd Higgs), $A$. The Yukawa couplings of $h$ and $A$ to down and up-type quarks in the T2HDM are:

$$
\begin{aligned}
h d \bar{d}: & Y_{d}^{S M} \times \tan \beta, \\
h u \bar{u}: & Y_{u}^{S M} \times \tan \beta ; u=u \text { or } c \\
h t \bar{t}: & Y_{t}^{S M} \times \cot \beta \\
A d \bar{d}: & Y_{d}^{S M} \times \tan \beta \gamma_{5}, \\
A u \bar{u}: & Y_{u}^{S M} \times \tan \beta \gamma_{5} ; u=u \text { or } c, \\
A t \bar{t}: & Y_{t}^{S M} \times \cot \beta \gamma_{5} .
\end{aligned}
$$

where $d=d, s$ or $b$ and $Y_{f}^{S M}=m_{f} / v, v=\sqrt{v_{1}^{2}+v_{2}^{2}}=$ $246 \mathrm{GeV}$.

We therefore see that while the Yukawa couplings of $h$ and $A$ to down quarks in the T2HDM have the same pattern as in the 2HDMII and the MSSM, i.e., $Y_{d} \propto$ $Y_{d}^{S M} \times \tan \beta$, their Yukawa couplings to a $u$ and a $c$ quark exhibit a different behavior, due to the special structure of the T2HDM's Yukawa matrices in (16). In particular, $Y_{c}$ and $Y_{u}$ are enhanced in the T2HDM by a factor of $\tan ^{2} \beta$ as compared to their values in the 2HDMII or in the MSSM, in which $Y_{u} \propto Y_{u}^{S M} \times \cot \beta$ for $u=u, c$ or $t$. Notice also that $Y_{t}$ (in the T2HDM) remains the same as in the 2HDMII and the MSSM.

Therefore, as will be shown below, since $\delta R^{M} \propto$ $\left(Y_{c} / Y_{b}\right)^{2}$ [see [11]], we can expect to have the following relations: $\delta R^{T 2 H D M} \sim \delta R^{S M} \propto\left(m_{c} / m_{b}\right)^{2}$ and $\delta R^{2 H D M I I}, \delta R^{M S S M} \ll \delta R^{T 2 H D M}$, when $\tan \beta$ is large. Note that in spite of the fact that $\delta R^{S M} \sim \delta R^{T 2 H D M}$, due to the small production rate of a SM (or a SM-like) Higgs via the gluon- $b$ and gluon- $c$ fusion channels, $\delta R^{S M}$ is overwhelmed by the very large background (i.e., $\delta R_{\exp }^{S M}$ ) and is thus unobservable.

In Tables IV and $\nabla$ we give the signal cross-sections at the Tevatron and at the LHC, respectively, for the T2HDM with $\tan \beta=30$ and evaluated at $\mu=m_{h}$ as the central value. To appreciate the uncertainties corresponding to variations of the factorization scale $\left(\mu_{F}\right)$,

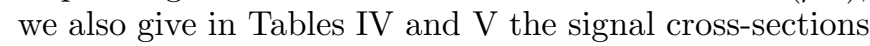
evaluated with $\mu_{F}=m_{h} / 4$ (upper uncertainty) and with $\mu_{F}=2 m_{h}$ (lower uncertainty), while keeping a fixed renormalization scale $\mu_{R}=m_{h}$. The effect of $\mu_{F}$ on the statistical significance of the signal will be discussed below.

All cross-sections in Tables IV and $\nabla$ are calculated with the set of cuts, as described in the previous section. ${ }^{[3]}$ To a good approximation, the signal crosssections given in Tables IV and $\nabla$ are essentially insensitive to whether the Higgs produced is $h$ or the pseudoscalar $A$. The difference between the two is the 1 versus the $\gamma_{5}$ couplings to fermions, which amounts to sign differences in the sub-leading terms involving $m_{b}$ or $m_{c}$.

Using eqs. (6)-(8) and (13), (14), we plot in Figs. 11 and2 the statistical significance $\left(N_{S D}\right)$ of $\delta R^{T 2 H D M}$ (the

[3] The relevant Lagrangian pieces of the T2HDM were implemented into COMPHEP. Also, the Higgs width was explicitly calculated within the T2HDM via COMPHEP. 

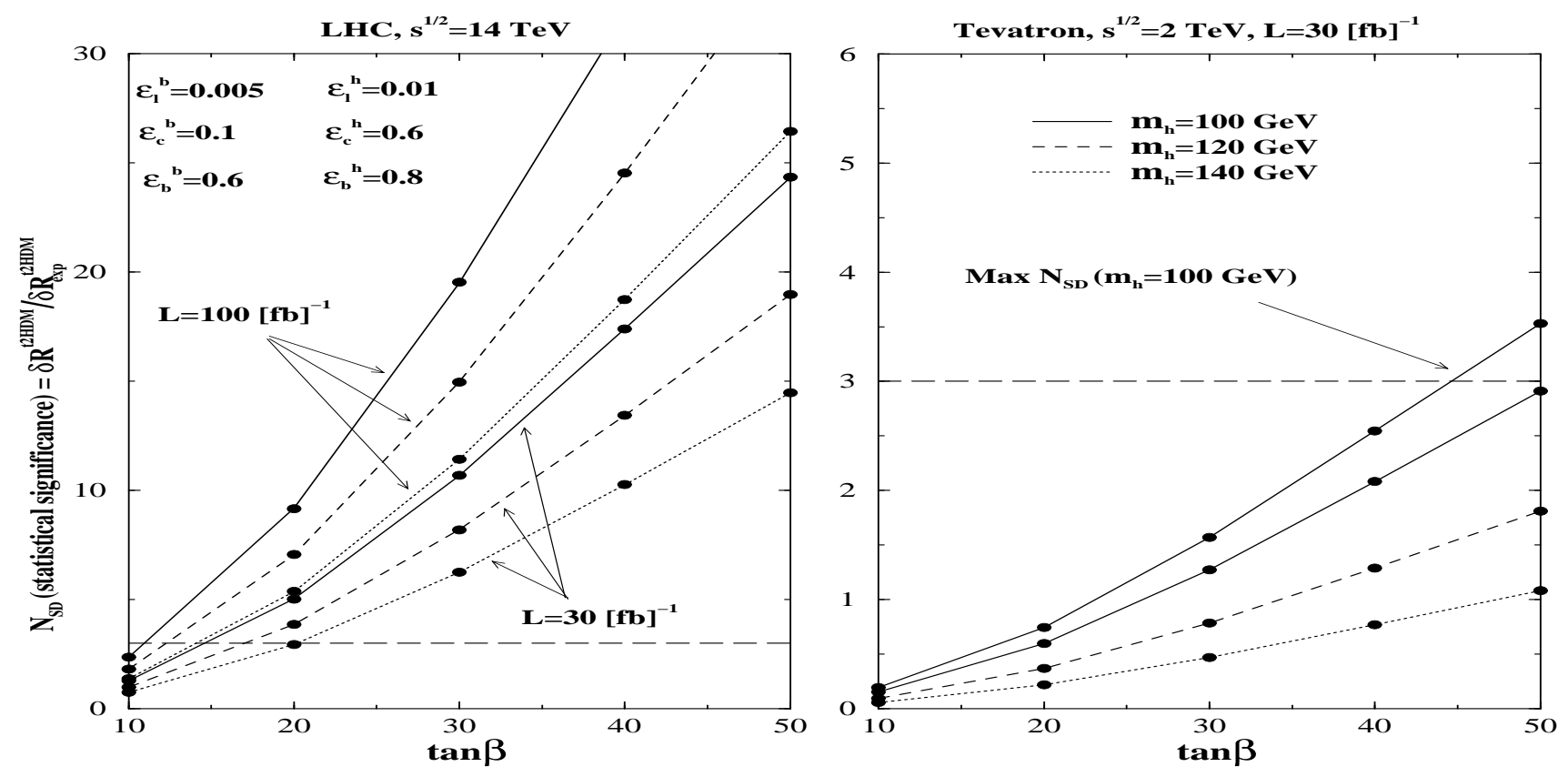

FIG. 1: The statistical significance of the signal in the T2HDM $\left(N_{S D}=\delta R^{T 2 H D M} / \delta R_{\text {exp }}^{T 2 H D M}\right)$ as a function of tan $\beta$ and for $m_{h}=100,120$ and $140 \mathrm{GeV}$, at the LHC with integrated luminosity of $30 \mathrm{fb}^{-1}$ and $100 \mathrm{fb}^{-1}$ (left plot) and at the Tevatron with integrated luminosity of $30 \mathrm{fb}^{-1}$ (right plot). The upper solid curve on the right plot is for the case of maximal charm and bottom $\overline{M S}$ quark masses at $\mu_{R}=m_{h}=100 \mathrm{GeV}$ (see discussion in section III). All cross-sections were calculated for $\mu=\mu_{F}=\mu_{R}=m_{h}$ and with the set of cuts 1-4 as outlined in section III

TABLE IV: Signal cross-sections in [fb], for the T2HDM with $\tan \beta=30$, at the Tevatron with a c.m. of $\sqrt{s}=2 \mathrm{TeV}$. All cuts are the same as for the background cross-sections at the Tevatron (see caption to Table III). The upper and lower uncertainties correspond to $\mu_{F}=m_{h} / 4$ and $\mu_{F}=2 m_{h}$, respectively.

Signal cross-sections for the T2HDM with $\tan \beta=30$

\begin{tabular}{|c||c|c|c|}
\hline$[\mathrm{fb}] \Downarrow /[\mathrm{GeV}] \Rightarrow$ & \multicolumn{3}{|c|}{ Tevatron, $\sqrt{s}=2 \mathrm{TeV}$} \\
\cline { 2 - 4 } & $m_{h}=100$ & $m_{h}=120$ & $m_{h}=140$ \\
\hline \hline$\sigma^{T 2 H D M}(g b \rightarrow b b b)$ & $970_{-9 \%}^{+9 \%}$ & $385_{-10 \%}^{+15 \%}$ & $166_{-12 \%}^{+20 \%}$ \\
$\sigma^{T 2 H D M}(g b \rightarrow b c c)$ & $42_{-9 \%}^{+9 \%}$ & $16.6_{-10 \%}^{+15 \%}$ & $7_{-12 \%}^{+20 \%}$ \\
$\sigma^{T 2 H D M}(g c \rightarrow c b b)$ & $72_{-14 \%}^{+35 \%}$ & $28.4_{-15 \%}^{+39 \%}$ & $12.1_{-15 \%}^{+42 \%}$ \\
& & & \\
$\sigma^{T 2 H D M}(g c \rightarrow c c c)$ & $3.1_{-14 \%}^{+35 \%}$ & $1.23_{-15 \%}^{+39 \%}$ & $0.52_{-15 \%}^{+42 \%}$ \\
\hline
\end{tabular}

signal within the T2HDM), as a function of $\tan \beta$ for the LHC with an integrated luminosity of $30 \mathrm{fb}^{-1}$ and 100 $\mathrm{fb}^{-1}$ and for the Tevatron with an integrated luminosity of $30 \mathrm{fb}^{-1}$. The statistical significance was calculated for $\tan \beta=10,20,30,40,50$ and interpolation was employed to obtain the results for other values of $\tan \beta$. As our nominal values, the following efficiency factors
TABLE V: Signal cross-sections in [fb], for the T2HDM with $\tan \beta=30$, at the LHC with a c.m. of $\sqrt{s}=14 \mathrm{TeV}$. All cuts are the same as for the background cross-sections at the LHC. See also captions to Table III and IV

\begin{tabular}{|c||c|c|c|}
\hline \multicolumn{3}{|c|}{ Signal cross-sections for the T2HDM with $\tan \beta=30$} \\
\hline$[\mathrm{fb}] \Downarrow /[\mathrm{GeV}] \Rightarrow$ & \multicolumn{3}{|c|}{$\mathrm{LHC}, \sqrt{s}=14 \mathrm{TeV}$} \\
\cline { 2 - 4 } & $m_{h}=100$ & $m_{h}=120$ & $m_{h}=140$ \\
\hline$\sigma^{T 2 H D M}(g b \rightarrow b b b)$ & $46000_{+1 \%}^{-18 \%}$ & $32400_{+2 \%}^{-12 \%}$ & $21900_{-1 \%}^{-9 \%}$ \\
$\sigma^{T 2 H D M}(g b \rightarrow b c c)$ & $2000_{+1 \%}^{-18 \%}$ & $1400_{+2 \%}^{-12 \%}$ & $940_{-1 \%}^{-9 \%}$ \\
$\sigma^{T 2 H D M}(g c \rightarrow c b b)$ & $3050_{-4 \%}^{+0 \%}$ & $2120_{-3 \%}^{+5 \%}$ & $1400_{-5 \%}^{+7 \%}$ \\
$\sigma^{T 2 H D M}(g c \rightarrow c c c)$ & $131_{-4 \%}^{+0 \%}$ & $92_{-3 \%}^{+5 \%}$ & $60_{-5 \%}^{+7 \%}$ \\
\hline
\end{tabular}

were chosen [1]: $\epsilon_{l}^{b}=0.005, \epsilon_{l}^{h}=0.01, \epsilon_{c}^{b}=0.1, \epsilon_{b}^{b}=$ $0.6, \epsilon_{b}^{h}=0.8$, with $\epsilon_{c}^{h}=0.6$ in Fig. 1 and $\epsilon_{c}^{h}=0.3$ in Fig. 2

Evidently, $\delta R^{T 2 H D M}$ can be easily observed at the LHC for $m_{h}=100-140 \mathrm{GeV}$, with a significance of more than 3-sigma for $\tan \beta \sim \mathcal{O}(20)$ if $\epsilon_{c}^{h}=0.6$ and for $\tan \beta \sim \mathcal{O}(35)$ if $\epsilon_{c}^{h}=0.3$. For example, already at the low luminosity stage of the LHC, i.e., with $L=30 \mathrm{fb}^{-1}$, a 5-sigma detection of $\delta R^{T 2 H D M}$ will be obtained for 

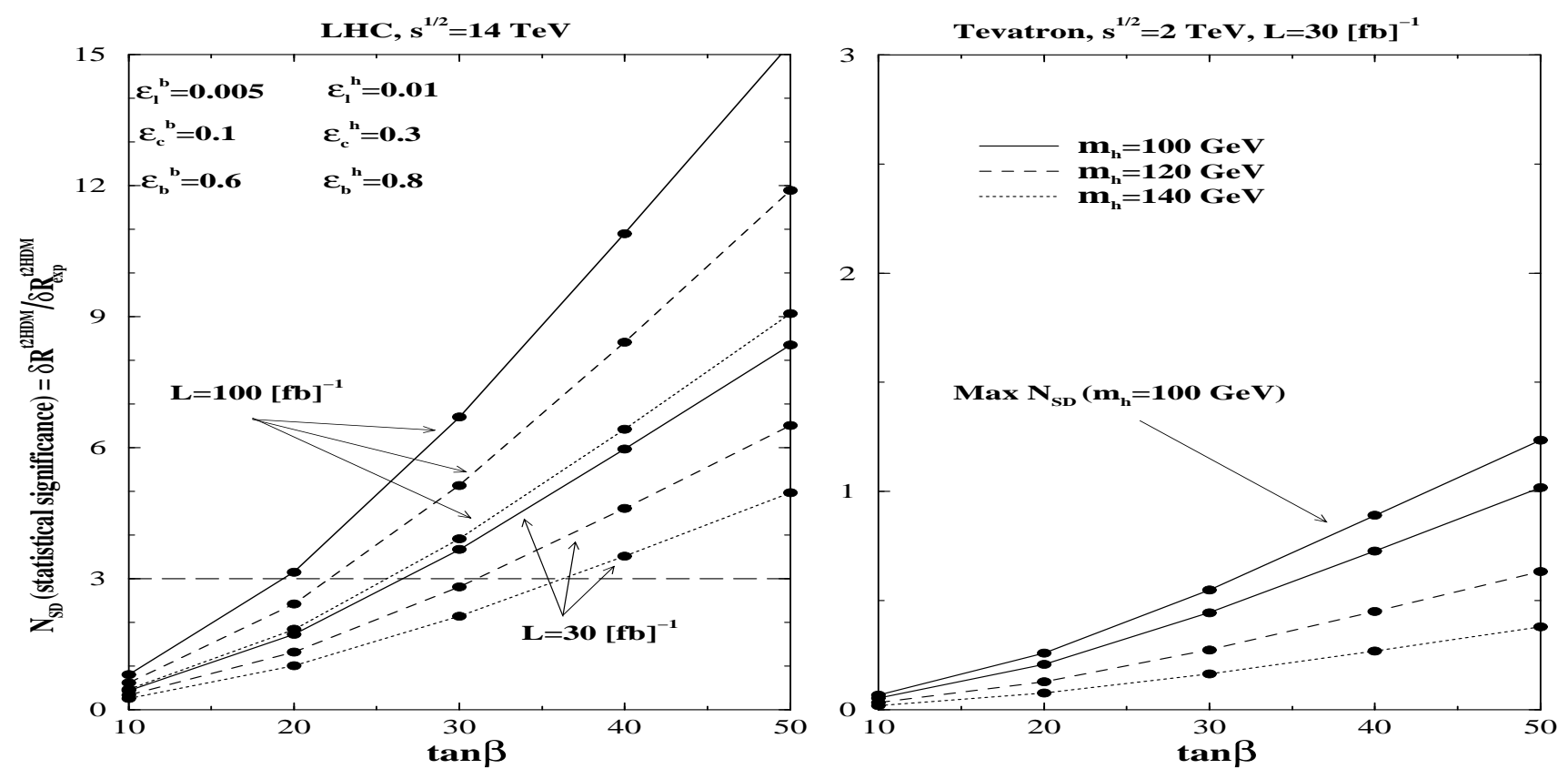

FIG. 2: Same as Fig. 1 for $\epsilon_{c}^{h}=0.3$.

$m_{h} \sim 100 \mathrm{GeV}$ if $\epsilon_{c}^{h}=0.6$ and $\tan \beta \sim 20$ or if $\epsilon_{c}^{h}=0.3$ and $\tan \beta \sim 35$. On the other hand, a 3-sigma detection of $\delta R^{T 2 H D M}$ will be possible at the Tevatron only if $\epsilon_{c}^{h} \gtrsim 0.5$ and if $m_{h}$ is around $100 \mathrm{GeV}$ and $\tan \beta \gtrsim 50$. The maximal signal case [obtained when $\bar{m}_{b}\left(m_{h}\right)$ and $\bar{m}_{c}\left(m_{h}\right)$ are evaluated with maximal $\bar{m}_{b}\left(\bar{m}_{b}\right)$ and $\bar{m}_{c}\left(\bar{m}_{c}\right)$ as initial conditions, see the discussion in section [II], is expected to give a 3-sigma effect at the Tevatron for $m_{h}=100 \mathrm{GeV}$ and $\tan \beta \gtrsim 45$ and if $\epsilon_{c}^{h} \sim 0.6$.

In Figs. 3 and 4 we give scatter plots which show the region in the $\epsilon_{b}^{h}-\epsilon_{c}^{h}$ plane that will allow a 3-sigma detection of $\delta R^{T 2 H D M}$ at the LHC when $\tan \beta=20$, $m_{h}=100-140 \mathrm{GeV}, \epsilon_{l}^{b}=0.005, \epsilon_{l}^{h}=0.01, \epsilon_{c}^{b}=0.1$, and with a $b$ jet tagging efficiency of $\epsilon_{b}^{b}=0.6$ in Fig. 3

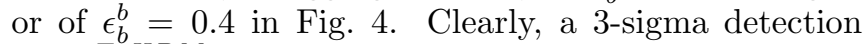
of $\delta R^{T 2 H D M}$ over the entire $m_{h}$ mass range shown, will be possible at the LHC for a b-tagging efficiency of $60 \%$ $\left(\epsilon_{b}^{b}=0.6\right)$ [1] and with $\epsilon_{b}^{h}, \epsilon_{c}^{h}$ as low as $\sim 0.3-0.4$ if $L=100 \mathrm{fb}^{-1}$, or with $\epsilon_{b}^{h}, \epsilon_{c}^{h} \sim 0.5-0.6$ if $L=30$ $\mathrm{fb}^{-1}$. Fig. 4 shows that for a lower $\mathrm{b}$ tagging efficiency of $\epsilon_{b}^{b}=0.4$, the requirements of $\epsilon_{b}^{h}, \epsilon_{c}^{h} \gtrsim 0.4$ for $L=100$ $\mathrm{fb}^{-1}$ and $\epsilon_{b}^{h}, \epsilon_{c}^{h} \gtrsim 0.6$ for $L=30 \mathrm{fb}^{-1}$, will also suffice for a 3 -sigma detection.

Finally, in Table VI we show the variation of the statistical significance of the signal $\left(N_{S D}\right.$ of $\left.\delta R^{T 2 H D M}\right)$ for different choices of the factorization scale, $\mu_{F}=m_{h} / 4, m_{h}$ and $2 m_{h}$. The numbers in Table VI correspond to the LHC with an integrated luminosity of $100 \mathrm{fb}^{-1}$ and are given for $\tan \beta=30$. Evidently, only mild changes (smaller than $5 \%$ ) in $N_{S D}$ are obtained when varying the factorization scale within the range $m_{h} / 4 \leq \mu_{F} \leq 2 m_{h}$.
TABLE VI: Comparison of the statistical significance in the T2HDM $\left(N_{S D}=\delta R^{T 2 H D M} / \delta R_{e x p}^{T 2 H D M}\right)$ for three values of the factorization scale $\mu_{F}=m_{h} / 4, m_{h}$ and $2 m_{h} . N_{S D}$ is given for $m_{h}=100,120$ and $140 \mathrm{GeV}$ and $\tan \beta=30$, at the LHC with an integrated luminosity of $100 \mathrm{fb}^{-1}$.

\begin{tabular}{|c||c|c|c|}
\hline \multicolumn{4}{|c|}{$N_{S D}$ in the T2HDM with $\tan \beta=30$} \\
\hline \multirow{\mathrm{GeV}}{*}{$\Rightarrow$} & \multicolumn{3}{|c|}{ LHC with $\mathcal{L}=100 \mathrm{fb}^{-1}$} \\
\cline { 2 - 4 } & $m_{h}=100$ & $m_{h}=120$ & $m_{h}=140$ \\
\hline \hline$\mu_{F}=m_{h} / 4$ & 6.37 & 5.15 & 4.03 \\
$\mu_{F}=m_{h}$ & 6.71 & 5.14 & 3.92 \\
$\mu_{F}=2 m_{h}$ & 6.51 & 5.03 & 3.77 \\
\hline
\end{tabular}

\section{B. The 2HDMII and the MSSM}

Let us now examine the expected signals $\delta R^{2 H D M I I}$ and $\delta R^{M S S M}$ in the 2HDMII and in the MSSM, respectively. Here too, we assume from the outset that $\tan \beta$ is large enough so that the non-standard Higgs can be observed in association with bottom quark jets at the hadron colliders under investigation (recall that if the neutral Higgs does not have the necessary $\tan \beta$ enhancement in its Yukawa coupling to the bottom quark, then our signal ceases to be effective). Thus, in what follows we will focus only on the large $\tan \beta$ case.

The 2HDMII Yukawa couplings follow from the 


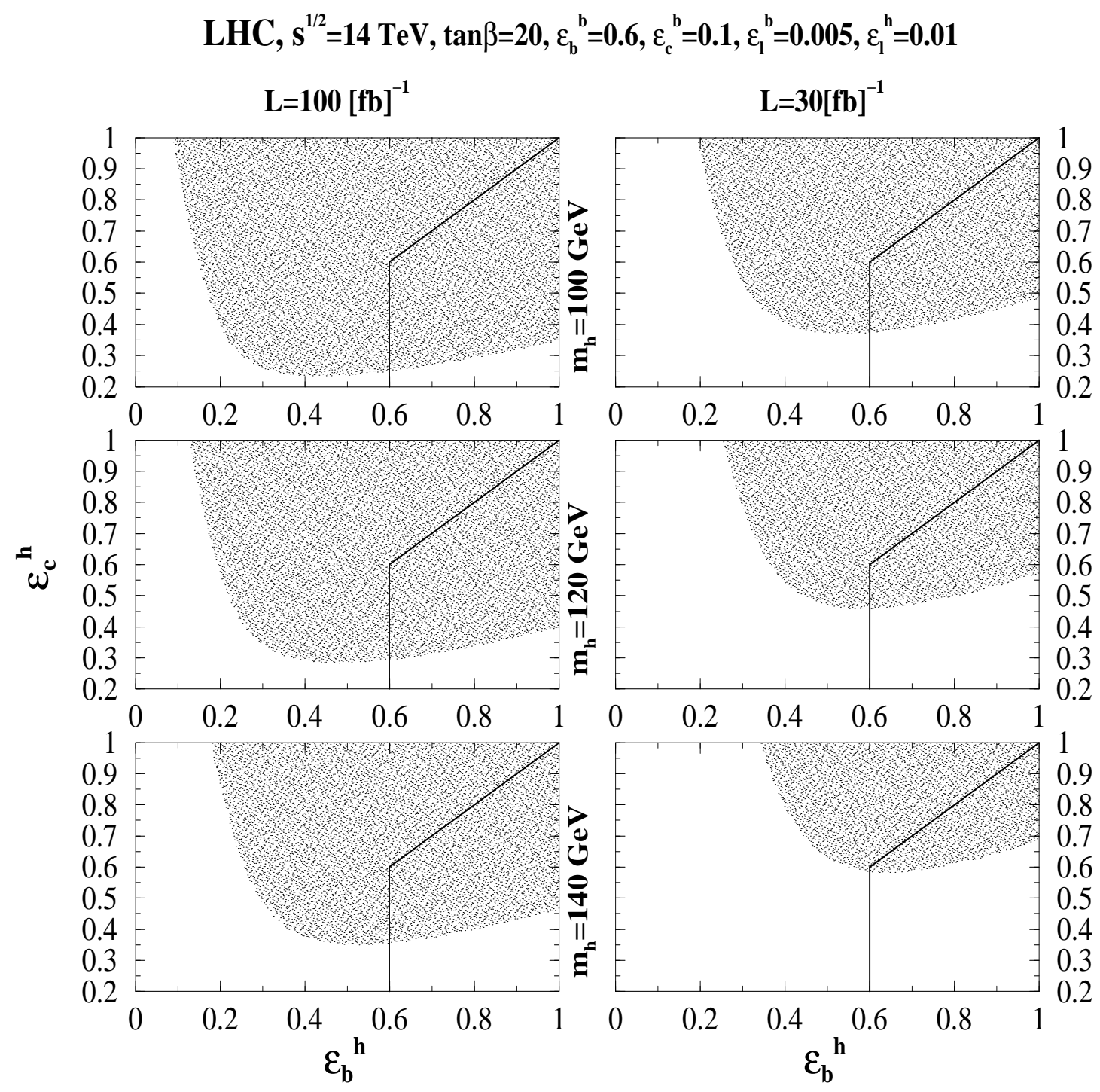

FIG. 3: Scatter plot for the LHC $(\sqrt{s}=14 \mathrm{TeV})$ in the $\epsilon_{b}^{h}-\epsilon_{c}^{h}$ plane, for $\tan \beta=20, \epsilon_{l}^{b}=0.005, \epsilon_{l}^{h}=0.01, \epsilon_{c}^{b}=0.1$ and $\epsilon_{b}^{b}=0.6$. The shaded area allows an above 3-sigma detection of $R^{T 2 H D M}$ at the LHC with integrated luminosity of $30 \mathrm{fb}^{-1}$ (right column) and $100 \mathrm{fb}^{-1}$ (left column) and for $m_{h}=100,120$ and $140 \mathrm{GeV}$ on the first, second and third rows, respectively. The shaded area enclosed to the right of the solid lines corresponds to the conditions $\epsilon_{b}^{h} \geq \epsilon_{c}^{h}$ and $\epsilon_{b}^{h} \geq \epsilon_{b}^{b}$. All cross-sections were calculated for $\mu=\mu_{F}=\mu_{R}=m_{h}$ and with the set of cuts 1-4 as outlined in section

Yukawa potential in (15) by setting $U^{1}=0$ and $D^{2}=0$. Thus, in this model $\Phi_{2}$ is responsible for the mass generation of the up-type quarks while the down-type quark masses are generated through the VEV of $\Phi_{1}$. Denoting again the three neutral Higgs species of the 2HDMII by $h, H$ (the two CP-even scalars) and $A$ (the CP-odd Higgs), their couplings to fermions are given by (see e.g., [13]):

$h d \bar{d}: \quad Y_{d}^{S M} \times[\sin (\beta-\alpha)-\tan \beta \cos (\beta-\alpha)]$, hū : $Y_{u}^{S M} \times[\sin (\beta-\alpha)+\cot \beta \cos (\beta-\alpha)]$, $H d \bar{d}: Y_{d}^{S M} \times[\cos (\beta-\alpha)+\tan \beta \sin (\beta-\alpha)]$, $H u \bar{u}: Y_{u}^{S M} \times[\cos (\beta-\alpha)-\cot \beta \sin (\beta-\alpha)]$, $A d \bar{d}: Y_{d}^{S M} \times \tan \beta \gamma_{5}$, 


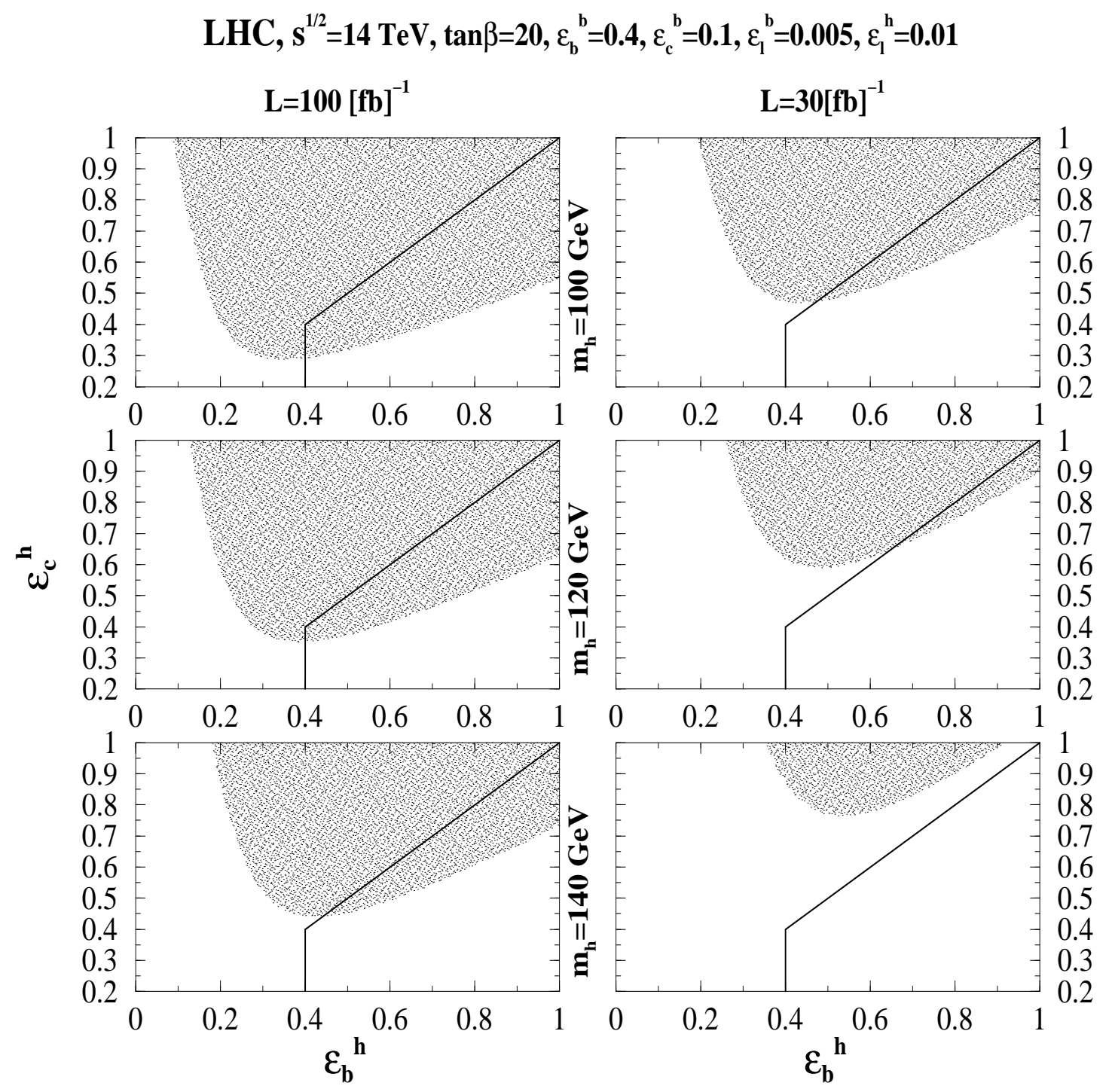

FIG. 4: Same as Fig. 3] for $\epsilon_{b}^{h}=0.4$.

$$
A u \bar{u}: Y_{u}^{S M} \times \cot \beta \gamma_{5},
$$

where $d(u)$ stands for a down(up) quark and $\alpha$ is the mixing angle in the CP-even Higgs sector. We will study three representative cases:

Case 1: $\cos (\beta-\alpha) \rightarrow 1$ or $\alpha \rightarrow \beta$.

Case $2: \cos (\beta-\alpha) \rightarrow 0$ or $\alpha \rightarrow \beta-\pi / 2$.

Case 3: $\cos (\beta-\alpha) \sim \sin (\beta-\alpha) \rightarrow 1 / \sqrt{2}$ or $\alpha \rightarrow \beta-\pi / 4$.

The charm and bottom Yukawa couplings and their ratio, $Y_{c} / Y_{b}$, are given in Table $\mathrm{VII}$ for the three cases above and for each of the neutral Higgs bosons $h, H$ and A.

The expected signals and their statistical significance for the three limiting cases and for the three neutral Higgs particles of the 2HDMII are compared to the T2HDM case in Table VIII Clearly, even in the more favorable cases in which $N_{S D}(2 H D M I I) \sim$ $N_{S D}(T 2 H D M) / \tan ^{2} \beta$, the expected statistical significance in the $2 \mathrm{HDMII}$ case is still at least two orders of magnitudes smaller than the one expected in the T2HDM when $\tan \beta \gtrsim 10$. Therefore, based on the results ob- 
TABLE VII: The charm and bottom Yukawa couplings $Y_{c}$ and $Y_{b}$ scaled by their SM Yukawa coupling $Y_{c}^{S M}$ and $Y_{b}^{S M}$, respectively, and their ratio $\left|Y_{c} / Y_{b}\right|$, in the $2 \mathrm{HDM}$ of type II. The Yukawa couplings of $h, H$ and $A$ are given for the three cases: (1) $\cos (\beta-\alpha) \rightarrow 1,(2) \cos (\beta-\alpha) \rightarrow 0$ and (3) $\cos (\beta-\alpha) \sim \sin (\beta-\alpha) \sim 1 / \sqrt{2}$, see also text.

\begin{tabular}{|c|c||c|c|c|}
\hline \multicolumn{5}{|c|}{$Y_{c}$ and $Y_{b}$ in the 2HDMII } \\
\hline Case & $\begin{array}{c}\text { Higgs } \\
\text { particle }\end{array}$ & $\mid Y_{c} / Y_{c}^{S M}$ & $\mid Y_{b} / Y_{b}^{S M}$ & $\left|Y_{c} / Y_{b}\right|$ \\
\hline \hline \multirow{2}{*}{1} & $h$ & $\frac{1}{\tan \beta}$ & $\tan \beta$ & $\frac{m_{c}}{m_{b} \tan ^{2} \beta}$ \\
& $H$ & 1 & 1 & $\frac{m_{c}}{m_{b}}$ \\
& $A$ & $\frac{1}{\tan \beta}$ & $\tan \beta$ & $\frac{m_{c}}{m_{b} \tan ^{2} \beta}$ \\
\hline \hline \multirow{2}{*}{2} & $h$ & 1 & 1 & $\frac{m_{c}}{m_{b}}$ \\
& $H$ & $\frac{1}{\tan \beta}$ & $\tan \beta$ & $\frac{m_{c}}{m_{b} \tan ^{2} \beta}$ \\
& $A$ & $\frac{1}{\tan \beta}$ & $\tan \beta$ & $\frac{m_{c}}{m_{b} \tan ^{2} \beta}$ \\
\hline \hline \multirow{3}{*}{3} & $h$ & $\frac{1}{\sqrt{2}}$ & $\frac{\tan \beta}{\sqrt{2}}$ & $\frac{m_{c}}{m_{b} \tan \beta}$ \\
& $H$ & $\frac{1}{\sqrt{2}}$ & $\frac{\tan \beta}{\sqrt{2}}$ & $\frac{m_{c}}{m_{b} \tan \beta}$ \\
& $A$ & $\frac{1}{\tan \beta}$ & $\tan \beta$ & $\frac{m_{c}}{m_{b} \tan ^{2} \beta}$ \\
\hline
\end{tabular}

tained for the T2HDM scenario, we conclude that, no signal of $R$ is expected to be observed at the LHC or at the Tevatron if the Yukawa Higgs sector is controlled by the 2HDMII. Hence, a measured signal of $\delta R$ at these hadron colliders will rule out the 2HDMII Higgs scenario with many standard deviations.

TABLE VIII: The expected 2HDMII signals, $\delta R^{2 H D M I I}$, and their statistical significance, $N_{S D}^{2 H D M I I}$, scaled by the corresponding values in the T2HDM, for cases $(1) \cos (\beta-\alpha) \rightarrow 1$, (2) $\cos (\beta-\alpha) \rightarrow 0$ and $(3) \cos (\beta-\alpha) \sim \sin (\beta-\alpha) \sim 1 / \sqrt{2}$, and for the three neutral Higgs of the model $h, H$ and $A$.

\begin{tabular}{|c|c|c|c|c|}
\hline \multicolumn{5}{|c|}{ Expected signals in the $2 \mathrm{HDMII}$} \\
\hline Case & $\begin{array}{l}\text { Higgs } \\
\text { particle }\end{array}$ & $\frac{\delta R^{2 H D M I I}}{\delta R^{T 2 H D M}}$ & $\frac{\delta R_{\exp }^{2 H D M I I}}{\delta R_{\exp }^{T 2 H D M}}$ & $\frac{N_{S D}^{2 H D M I I}}{N_{S D}^{T} 2 H D M}$ \\
\hline 1 & $\begin{array}{l}h \\
H \\
A\end{array}$ & $\begin{array}{c}\frac{1}{\tan ^{4} \beta} \\
1 \\
\frac{1}{\tan ^{4} \beta} \\
\end{array}$ & $\begin{array}{c}1 \\
\tan ^{2} \beta \\
1 \\
\end{array}$ & $\begin{array}{l}\frac{1}{\tan ^{4} \beta} \\
\frac{1}{\tan ^{2} \beta} \\
\frac{1}{\tan ^{4} \beta}\end{array}$ \\
\hline 2 & $\begin{array}{l}h \\
H \\
A\end{array}$ & $\begin{array}{c}1 \\
\frac{1}{\tan ^{4} \beta} \\
\frac{1}{\tan ^{4} \beta} \\
\end{array}$ & $\begin{array}{c}\tan ^{2} \beta \\
1 \\
1\end{array}$ & $\begin{array}{l}\frac{1}{\tan ^{2} \beta} \\
\frac{1}{\tan ^{4} \beta} \\
\frac{1}{\tan ^{4} \beta} \\
\end{array}$ \\
\hline 3 & $\begin{array}{l}h \\
H \\
A\end{array}$ & $\begin{array}{l}\frac{1}{\tan ^{2} \beta} \\
\frac{1}{\tan ^{2} \beta} \\
\frac{1}{\tan ^{4} \beta}\end{array}$ & $\begin{array}{l}1 \\
1 \\
1\end{array}$ & $\begin{array}{l}\frac{1}{\tan ^{2} \beta} \\
\frac{1}{\tan ^{2} \beta} \\
\frac{1}{\tan ^{4} \beta}\end{array}$ \\
\hline
\end{tabular}

The 2HDMII Higgs framework also underlies the MSSM Higgs sector. However, due to the supersymmetric structure of the theory, the MSSM's Higgs sector is completely determined at tree-level by only two free parameters, conventionally chosen to be $m_{A}$ and $\tan \beta$. That is, the mixing angle $\alpha$ is fixed by $m_{A}$ and $\tan \beta$ at tree level. Concentrating again on the large $\tan \beta$ case, in the MSSM one can distinguish two limiting cases:
Case 1: $m_{A} \lesssim m_{h}^{\max }$, where $m_{h}^{\max } \sim 120$ or $135 \mathrm{GeV}$, is the maximal allowed mass of the lighter CP-even Higgs after radiative corrections are included in the CP-even sector, depending whether one takes the minimal or the maximal stop mixing scenario, respectively [14]. In this case $\cos (\beta-\alpha) \rightarrow 1$.

Case 2: $m_{A}^{2}>>m_{Z}^{2}$, the so called decoupling limit. In this limit $\cos (\beta-\alpha) \rightarrow 0$.

These two MSSM limiting cases for large $\tan \beta$ remain valid also after the radiative corrections to the Higgs sector are included [15], thereby shifting the value of the mixing angle $\alpha$ (the higher order contribution to $\alpha$ can, on the other hand, cause a large shift to the lighter CPeven Higgs mass, $\left.m_{h}\right)$. ${ }^{[4]}$ It should also be noted that, in the large $\tan \beta$ case, $\cos ^{2}(\beta-\alpha)$ approaches zero very rapidly as $m_{A}$ is increased. In particular, an order of magnitude drop of $\cos ^{2}(\beta-\alpha)$ (from 1 to 0.1 ) is spanned over no more than about $10 \mathrm{GeV}$ mass range of $m_{A}$ [15].

Since cases 1 and 2 in the MSSM Higgs sector are equivalent to cases 1 and 2 of the 2HDMII framework, they have the same Yukawa couplings pattern as given in Table VII Thus, applying the results obtained in the $2 \mathrm{HDMII}$ to the MSSM case, we conclude here too that $\delta R^{M S S M}$ cannot be observed either at the Tevatron or at the LHC. Reversing the argument, a measured signal of $\delta R$ at these hadron colliders will rule out the MSSM.

\section{SUMMARY}

To summarize, we have proposed a signal, $\delta R$ [see (11)], based on counting the number of three heavy $(c$ or $b)$ jets events versus the number of events with three $b$ jets, in processes in which the neutral Higgs is produced in association with a single high $p_{T} c$ or $b$ jet. This signal assumes that the Higgs Yukawa coupling to the $b$ quark is enhanced, say by a large $\tan \beta$ in multi Higgs doublet models, and that the neutral Higgs will therefore be observed in association with $b$ jets at future hadron collider experiments.

This signal was calculated in the framework of multi Higgs models which have at least one neutral Higgs with an enhanced Yukawa coupling to the $b$ quark when $\tan \beta$ is large. We have found that in such cases, the signal to background cross-section ratio is typically (after applying some useful kinematical cuts) $S / B \sim 0.1-1$ at the LHC and $S / B \sim 0.05-0.3$ at the Tevatron, depending on the value of $\tan \beta$.

The measurement of such a signal will require an efficiency for distinguishing a $c$ jet from a light jet at the level

[4] There are also vertex corrections to the Higgs-fermion Yukawa couplings which do not depend on the mixing angle $\alpha$. However, these have a negligible effect in the up quark sector, and even more so for large $\tan \beta$ values [16]. 
of about $\epsilon_{c}^{h} \sim 20 \%-30 \%$ or $\epsilon_{c}^{h} \sim 50 \%-60 \%$ at the LHC or at the Tevatron, respectively. Such efficiencies seem to be somewhat higher than what has been attained in the simulations to date $\left(\epsilon_{c}^{h} \sim 10 \%\right)$ 17]. We have shown that if such $c$ jet tagging efficiencies are acomplished, then our signal will be very efficient for probing the ratio between the charm and bottom Yukawa couplings, $Y_{c} / Y_{b}$, thus allowing a deeper insight into the Higgs Yukawa potential. This, in turn, will be useful for categorizing the theory that underlies the Higgs sector. It should be noted, that our predictions for the Tevatron rely on an accumulated yearly luminosity of 30 inverse [fb], which is, at present, considerably higher than the expected Fermilab's run II luminosity.

It should also be noted that the background estimate made for our signal was based on a low probability for misidentifying a light jet as a heavy jet, i.e., $\epsilon_{l}^{h} \sim \mathcal{O}(1 \%)$. While that value is comparable to the present estimate for $\epsilon_{l}^{b}$ (or even a little conservative), it is somewhat optimistic for $\epsilon_{l}^{c}$ which is not yet well studied by present detector simulations. A more realistic value for the distinction between a light jet and a charm jet, based on the technology of today, would be $\epsilon_{l}^{c} \sim \mathcal{O}(10 \%)$. Nontheless, our background estimate (and therefore also our estimate for the significance of the signal) holds roughly for values of $\epsilon_{l}^{c}$ not larger than about $10 \%$. For $\epsilon_{l}^{c}$ at the level of tens of percents there may well be other processes, e.g., $g g \rightarrow g g g$, that may alter our signal to background estimate to the level that the observability of our signal becomes difficult. Another potential problem to the detection of our signal may arise if the trigger algorithm for b-jet tagging requires a minimum transverse momentum cut at the level of $100 \mathrm{GeV}$ and above and the $p_{T}$ cut of $30 \mathrm{GeV}$ we used for the LHC and $15 \mathrm{GeV}$ for the Tevatron proves to be too optimistic. In that case also, the signal proposed in this paper may be degraded and difficult to observe due to the large QCD background.

If the difficulties mentioned above can be surmounted and such a signal is detected at the Tevatron or at the LHC, then the popular MSSM and the 2HDMII can be ruled out with many standard deviations, since in these theories the ratio $Y_{c} / Y_{b}$ is too small for our signal to have any measurable consequences. On the other hand, we have shown that within a version of a $2 \mathrm{HDM}$ - the "2HDM for the top" (T2HDM) - in which the large mass of the top quark is naturally accommodated for large $\tan \beta$, our signal can be easily observed at the LHC within the entire relevant mass range of the neutral Higgs if $\tan \beta \gtrsim 20$ and $\epsilon_{c}^{h} \sim 20 \%-30 \%$. In addition, if the neutral Higgs of the T2HDM has a mass around $100 \mathrm{GeV}$ and $\tan \beta \gtrsim 50$, then our signal may also give an effect with more than 3-sigma significance at the Tevatron provided that $\epsilon_{c}^{h} \sim 50 \%-60 \%$.

\section{Acknowledgments}

We would like to thank F. Paige, W. Kilgore and S. Dawson for their helpful comments. G.E. also thanks the Technion President Fund. This work was also supported in part by US DOE Contract Nos. DE-FG02-94ER40817 (ISU) and DE-AC02-98CH10886 (BNL).
[1] See e.g., D. Cavalli et al., in "Les Houches 2001, Physics at TeV Colliders", R. Aurenche et al., IN2P3, Paris, France (2001), pp. 1-120, hep-ph/0203056 A. Djouadi, review talk given at the 7 th Workshop on High-Energy Physics Phenomenology, WHEPP VII, Allahabad, India, January 4-15, 2002, Pramana 40, pp. 215-238 (2003), hep-ph/0205248 B. Mellado, for the CMS and ATLAS collaborations, talk given at the 14th Topical Conference on Hadron Collider Physics (HCP 2002), Karlsruhe, Germany, September 29 - October 4, 2002, Springer (2003), pp. 348-355, hep-ex/0211062

[2] D. Dicus, T. Stelzer, Z. Sullivan and S. Willenbrock, Phys. Rev. D59, 094016 (1999); F. Maltoni, Z. Sullivan and S. Willenbrock, Phys. Rev. D67, 093005 (2003); R.V. Harlander and W.B. Kilgore, hep-ph/0304035

[3] J. Campbell, R.K. Ellis, F. Maltoni and S. Willenbrock, Phys. Rev. D67, 095002 (2003).

[4] C.S. Huang and S.H. Zhu, Phys. Rev. D60, 075012 (1999); J.-J. Cao, G.-P. Gao, Y.-L. Su and J.M. Yang, Phys. Rev. D68, 075012 (2003);

[5] J. Dai, J.F. Gunion and R. Vega, Phys. Lett. B345, 29 (1995); J. Dai, J.F. Gunion and R. Vega, Phys. Lett. B387, 801 (1996); E. Richter-Was and D. Froidevaux, Z. Phys. C76, 665 (1997); J.L. Diaz-Cruz, H.J. He, T. Tait and C.P. Yuan, Phys. Rev. Lett. 80, 4641 (1998); C. Balazs et al., Phys. Rev. D59, 055016 (1999); M. Carena,
S. Mrenna and C.E. Wagner, Phys. Rev. D60, 075010 (1999).

[6] D. Choudhury, A. Datta and S. Raychaudhuri, hep-ph/9809552

[7] Comphep version 4.2.0 - a package for evaluation of Feynman diagrams and integration over multi-particle phase space. User's manual for version 3.3: A. Pukhov et al., hep-ph/9908288 URL: http://theory.sinp.msu.ru/comphep

[8] H.L. Lai et al., Eur. Phys. J. C12, 375 (2000).

[9] See e.g., J.A.M. Vermaseren, S.A. Larin and T. van Ritbergen, Phys. Lett. B405, 327 (1997); H. Fusaoka and Y. Koide, Phys. Rev. D57, 3986 (1998).

[10] K. Hagiwara et al. (Partical Data Group), Phys. Rev. D66, 010001 (2002).

[11] See e.g., E. Richter-Was, D. Froidevaux and L. Poggioli, ATLAS Peport No. ATL-PHYS-98-131; M. Sapinski (on behalf on the ATLAS collaboration), Eur. Phys. J. directC 4S1, 08 (2002).

[12] A.K. Das and C. Kao, Phys. Lett. B372, 106 (1996); K. Kiers, A. Soni and G.-H. Wu, Phys. Rev. D59, 096001 (1999); G.-H. Wu, and A. Soni, Phys. Rev. D62, 056005 (2000); K. Kiers, A. Soni and G.-H. Wu, Phys. Rev. D62, 116004 (2000).

[13] For reviews on the Higgs sector in the Standard Model, the two Higgs doublet model and in supersymmetry, see 
e.g., J. Gunion, H. Haber, G. Kane and S. Dawson, "The Higgs Hunters Guide", Addison-Wesley, Reading 1990; D. Atwood, S. Bar-Shalom, G. Eilam and A. Soni, Phys. Rep. 347, 1 (2001).

[14] See e.g., J.R. Espinosa and R.-J. Zhang, Nucl. Phys. B586, 3 (2000); S. Heinemeyer, W. Hollik and G. Weiglein, hep-ph/0002213
[15] See e.g., J.F. Gunion, H.E. Haber and R. van Kooten, hep-ph/0301023 and references therein.

[16] See e.g., M. Carena, H.E. Haber, H.E. Logan and S. Mrenna, Phys. Rev. D65, 055005 (2002) and references therein.

[17] F. Paige and W. Kilgore, private communications. 